\title{
Utilisation énergétique des aquifères profonds Contraintes et défis de la nouvelle géothermie
}

\author{
par Jean-Claude Vathaire \\ de Geotherma SA
}

\section{HISTORIQUE}

Si l'invention de l'exploitation géothermale reste une idée française (le baron Lardarel, 1904, Lardarello/Italie) et après une première tentative Dogger en 1962 à Carrières-surSeine, il faut attendre 1964 pour voir réaliser à Paris la première utilisation énergétique d'une ressource aquifère profonde. L'aquifère utilisé, l'Albien, est à une profondeur de 600 mètres et sa température est de $27^{\circ} \mathrm{C}$. Si l'Albien produit une eau de très bonne qualité, le recours à des aquifères plus profonds, plus chauds, salés, exigent une technologie plus élaborée. En 1969, P. Maugis à Melun l'Almont, expérimentait pour la première fois la technologie du doublet, l'eau produite par un premier puits était réinjectée par un second puits suffisamment éloigné après récupération de l'énergie en surface au moyen d'un échangeur. Melun l'Almont fut mis en exploitation en 1971.

De 1971 à 1981, seules quelques opérations furent réalisées, mais le véritable démarrage eut lieu en 1981.

Au total quatre-vingts opérations basse-énergie furent réalisées avec des succès divers, cinquante-sept dans le Bassin Parisien, quinze dans le Bassin Aquitain, treize dans des régions diverses. Dans le Bassin Parisien, on dénombre un seul échec (Provins) et trois succès partiels, en Aquitaine, un échec et cinq succès partiels. Dans les autres régions, douze tentatives furent des échecs et une seule un succès partiel (fig. I).

La géothermie peut être définie simplement comme

\begin{abstract}
l'exploitation commerciale de la chaleur stockée dans l'écorce terrestre et ayant pour origine la radioactivité naturelle des roches et les échanges thermiques avec les zones plus profondes. Le potentiel théoriquement disponible est considérable, un kilomètre carré sur dix kilomètres de profondeur représenterait une énergie équivalente à quinze millions de TEP (tonnes équivalent-pétrole).
\end{abstract}

Mais cette énergie n'est récupérable que si le sous-sol contient à la profondeur voulue, un aquifere exploitable. Selon les formations géologiques et les profondeurs où se situent les aquifères, plusieurs niveaux de ressources géothermales ont été différenciés :

- géothermie haute énergie correspondant à des ressources dont la température est supérieure à $150^{\circ} \mathrm{C}$ et directement exploitable pour la production d'électricité,

- géothermie moyenne énergie, à des ressources dont la temperature se situe entre $90 \mathrm{et} 150{ }^{\circ} \mathrm{C}$ et valorisable indirectement pour la production d'électricité en recourant à des fluides à basse température de vaporisation tournant en circuit fermé (cycle de Rankine),

- géothermie basse énergie, à des ressources dont la température se situe entre 25 et $90^{\circ} \mathrm{C}$ et directement utilisable pour des applications thermiques,

- géothermie très basse énergie, à des ressources peu profondes, généralement à moins de cents mètres de profondeur et indirectement utilisables pour des applications thermiques au moyen de pompes à chaleur.

In France, the urilisation of low and very low energy geothermic resource kenw a quick growih, mainly at the end of the second oil crisis. This development mainly occured between 1980 and 1986 in a posime context in which the factor of the high cost of fossil energies plaved an impontant role.

From 1986 onvard techmical, economical and structural difficulties have hampered its development. The great majority of the operations which were carried out at that time is now effective, thanks to the adminis tration, the researeh deparments and the industrial sector concerned which enabled them to overcome the difficulties.

According to the actual econmic context, the perspectives of a development similar to the 1980-1986 one in France are low but there are real possibilities as to the optimisation and the nodelling of equipments and the diversification of the utilsations. The recent realisations are confiming lhis potentialit: 


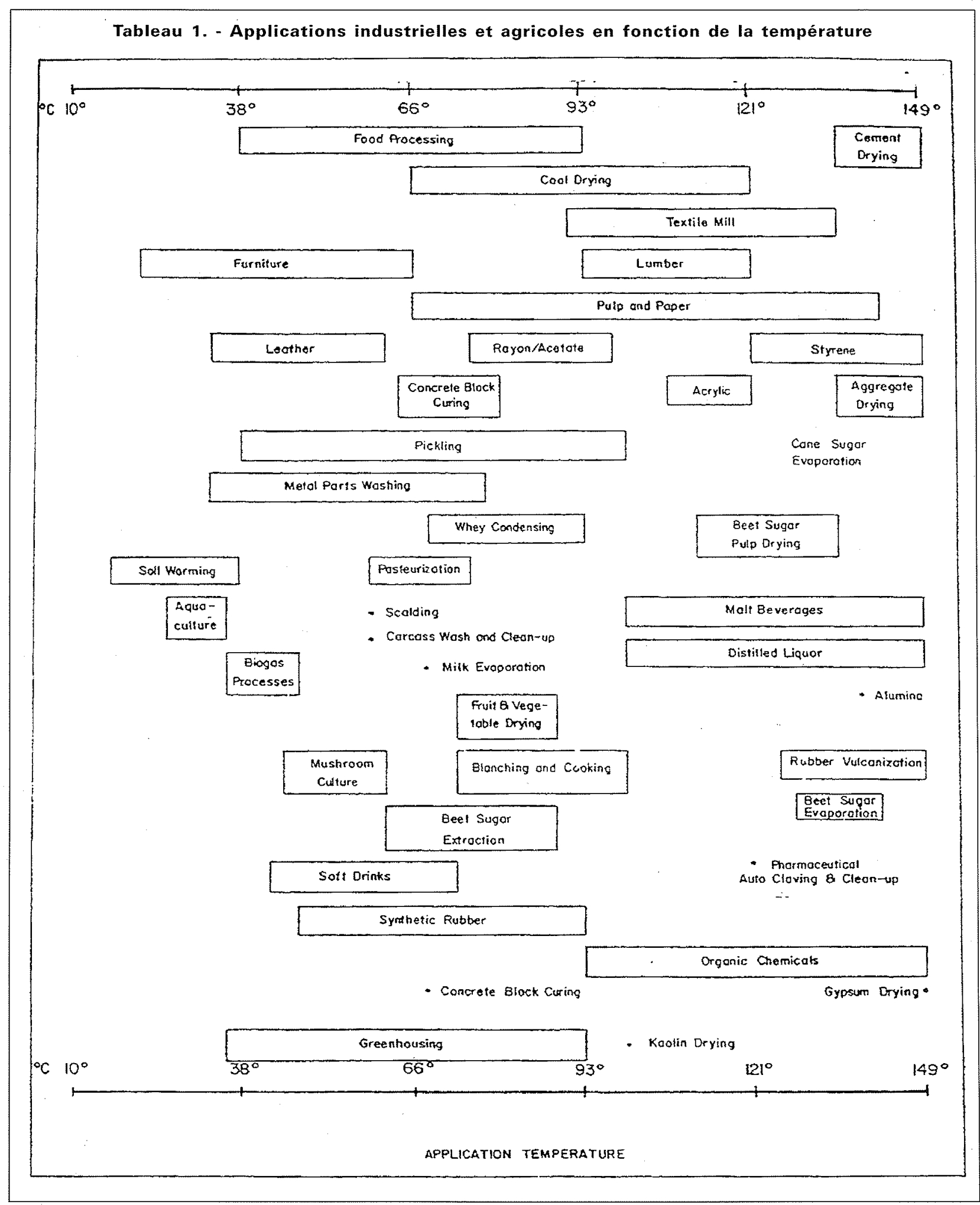

\subsection{Haute énergie}

La première exploitation commerciale date de 1904 (Lardarello - Italie). Avant même que nos sociétés connaissent le premier choc pétrolier (1973), près de quarante pays procédaient à l'exploitation de telles ressources pour la production d'électricité. Les pays concernés jalonnent les grands arcs volcaniques: Etats-Unis, Japon, Amérique Centrale, Amérique Latine, Philippines, Indonésie, Nouvelle Zélande, Islande, Italie, etc...

Aujourd'hui, 350 installations dont la taille va de quelques MWe à plusieurs dizaines de MWe, représentent une puissance installée de près de $6800 \mathrm{MWe}$. Le plus gros producteur est, de loin, les USA avec plus de $2800 \mathrm{MWe}$ 
installés, suivis des Philippines (1 227), du Mexique (753), de l'Italie (631), du Japon (414), de l'Indonésie (310), de la Nouvelle Zélande (286), etc...

L'Union Européenne ne représente que 9,4\% de cette production, assurée par l'Italie, la France (Antilles, 4,2 MWe), et le Portugal (Açores, 4 MWe).

Durant la dernière décennie, la progression de la hauteénergie a été considérable $(+43 \%)$. On prévoit un nouvel essor de $45 \%$ à l'an 2000 et de $90 \%$ à l'an 2010. Concernant l'Union Européenne, seule l'ltalie envisage de développer ce type de production ( $+35 \%$ à l'an 2000$)$.

\subsection{Moyenne énergie}

La géothermie moyenne énergie représente environ $400 \mathrm{MWe}$ installés de par le monde, produits par moins d'une centaine de centrale à cycle de Rankine. Son développement est relativement récent, mettant en cuvre depuis 1980, une technologie plus complexe, maîtrisée essentiellement par les Américains et les Israéliens. La puissance unitaire de ces installations va de quelques KWe à quelques MWe.

Les perspectives de développement sont réelles car la technologie actuelle permet de produire un KWe électrique à un coût attractif avec des équipements standardisés sur catalogue, montés le plus souvent sur Skids

Le potentiel disponible correspond à des zones géographiques plus vastes qu'en haute énergie, mieux réparties de par le monde, mais plus localisées (généralement aquifères à fort gradient géothermique)

\subsection{Basse énergie}

La géothermie basse énergie est certainement celle qui a connu le développement le plus important, avec une accélération particulièrement nette à la sortie du deuxième choc pétrolier (1980). Elle est activement exploitée dans plus de 40 pays représentant une puissance installée à l'échelle mondiale de l'ordre de $8230 \mathrm{MWth}$.

Le développement de l'utilisation de ces ressources a été particulièrement rapide de 1980 à 1985, puis s'est accru beaucoup moins rapidement de 1985 à 1995 (14\%), en raison essentiellement de la compétition du prix des énergies conventionnelles.

Les principaux utilisateurs de ce type d'énergie sont principalement et dans l'ordre, la Chine (I $915 \mathrm{MWt}$ ), les U.S.A. (1 $874 \mathrm{MWt}$ ), l'Islande (1 $443 \mathrm{MWt}$ ), la Hongrie (340 MWt), la France (337 MWt), le Japon (318 MWt), l'Italie (307 MWt), la Nouvelle Zélande (264 MWt), la Russie $(210 \mathrm{MWt})$, la Turquie (140 MWt), la Roumanie (130 MWt), etc...

Pour sa part l'Union Européenne représente plus de $8 \%$ de la production mondiale dont plus de $4 \%$ pour le territoire français. Cette production d'énergie à partir d'aquifères profonds représente une économie annuelle de l'ordre de 310000 TEP.

Selon différentes sources, l'exploitation commerciale des ressources géothermales basse énergie devrait comnaître dans les années à venir, un développement relativement important. En corrigeant certains chiffres nettement surévalués au cours des années précédentes, et en prenant un taux de progression de l'ordre de $10 \%$ (Fridleifsson \& Freeston, 1990), la puissance mondiale installée serait d'environ $13000 \mathrm{MWt}$ à l'an 2000 et de $34000 \mathrm{MWt}$ en 2010, ce développement devrait surtout s'effectuer dans les Pays d'Europe Centrale et Orientale, la Russie et en Chine.

\subsection{Très basse énergie}

L'utilisation de ressources dont la température est inférieure à $25^{\circ} \mathrm{C}$ (très basse énergie), permet de couvrir des besoins en chauffage et/ou en refroidissement à l'aide de Pompes à Chaleur alimentées par des aquifères situés en général à moins de 100 mètres de profondeur.

Ce créneau vise essentiellement les logements neufs et le tertiaire et connaît depuis quelques années un essor important, principalement dans le Nord de l'Europe, au Japon et aux USA, aussi dans ce pays le nombre de PAC est-il passé de 25000 en 1989 à 150000 en 1992. Actuellement la part de la très basse énergie représente plus de $75 \%$ de la puissance installée aux USA (1 444 MWth 1874).

Selon les références américaines, l'utilisation de ces ressources permet de réduire de $72 \%$ la consommation énergétique par rapport à une installation classique de conditionnement d'air faisant appel uniquement à l'électricité et permet de répondre aux trois objectifs majeurs de National Energy Strategy : limiter la demande en électricité, préserver l'environnement et économiser les énergies fossiles. On considère que dans les années à venir les PAC sur nappe souterraine pourraient à terme aux USA remplacer partiellement les PAC sur air (850 000 en service en 1989).

\section{II — CONTRAINTES LIÉES À L'EXPLOI- TATION DE LA GÉOTHERMIE}

Au niveau de l'exploitation, on constate alors que tout bassin sédimentaire ne se prédispose pas forcément à une exploitation géothermique qui doit assurer débit et température suffisante. A ce titre il apparaît alors, de même que pour les autres ressources minières, une notion de gisement dont les caractéristiques de perméabilité et de température permettront de définir l'exploitabilité de la ressource. En particulier les explorations menées en Alsace, en Bresse, en Limagne, dans le couloir rhodanien et en Languedoc se sont révélées négatives malgré l'importance des bassins sédimentaires et la présence d'aquifères profonds.

Un autre paramètre intervient au niveau de l'exploitabilité géothermique d'un aquifère profond, à savoir les caractéristiques propres du fluide géothermal qui peuvent créer des contraintes limitant ou voire condamnant l'exploitation de la ressource. C'est le cas plus particulièrement pour le Dogger du Bassin Parisien (profondeur 1700 à $2000 \mathrm{~m}$, température 60 à $85^{\circ} \mathrm{C}$, débit de 150 à $300 \mathrm{~m}^{3} / \mathrm{h}$, salinité 15 à $38 \mathrm{~g} / \mathrm{l}$ ). Les opérations du Bassin Aquitain, qui elles, exploitent des eaux peu minéralisées n'ont pas connu de problème majeur.

Ces difficultés liées à la profondeur du Dogger, présents dès le forage de Carrières-sur-Seine en 1962, sembleraient avoir été surmontées avec la technologie innovée en 1969 à Melun l'Almont; et la majeure partie des opérations furent réalisées dans un laps de temps relativement court, entre 1982 et 1985 sans avoir été confronté à de grandes difficultés de mise en œuvre.

Or le fluide géothermal du Dogger contient des sels (essentiellement $\mathrm{Cl} \mathrm{Na}$ ) et des gaz dissous $\left(\mathrm{CO}_{2}, \mathrm{H}_{2} \mathrm{~S}\right)$ dans des teneurs variables suivant la localisation gếographique, ainsi qu'une activité bactérienne importante (BSR) sur certains sites.

Le pouvoir corrosif de cette eau avait été pressenti dès 1969 et si en surface il avait été préconisé des échangeurs en titane, il paraissait difficile de recourir, pour des raisons économiques, à des matériaux adaptés pour les tubages. Certes TOTAL, en 1976, avait réalisé un doublet équipé de tubages à fibre de verre, mais la plupart des matériaux utilisés restaient de type classique, pompe, chambre de pompage en gros diamètre.

L'opération de Carrières-sur-Seine et de Villeneuve-laGarenne se révélaient être techniquement et économiquement des demi-succès. GEOTHERMA mettait en œuvre en 1977, au Mée-sur-Seine, une autre technologie utilisant un gel thixotropique étanche et diélectrique en lieu et place du 
ciment dans l'annulaire tubage-terrain, permettant ainsi à terme la récupération du tubage corrodé et son remplacement.

Mais la pression politique et économique de l'époque (1981-1982) était telle que ces réponses techniques aux problèmes technologiques, n'avaient pas de suite en raison de leur prix et de leur mise en ouvre délicate. La majeure partie des opérations furent donc réalisées avec des tubages classiques en acier K 55 étiré sans soudure.

\subsection{Difficultés}

Dès 1983-1984, apparurent des difficultés: phénomènes de corrosion-dépôt, plus ou moins conséquents selon les teneurs en sels dissous, notamment en sulfures, perturbant plus ou moins gravement l'exploitation des installations.

Parallèlement, apparaissaient des problèmes économiques liés à la baisse du coût des énergies fossiles sur lequel étaient généralement indexées les recettes. Financées par emprunt ces opérations se sont retrouvées avec un différentiel important entre les taux d'intérêt en vigueur en 1980 et le taux d'inflation effectif des années 1985-1990. Apparaissent également des problèmes de structure, la majeure partie des maîtres d'ouvrage étaient des collectivités locales ou des entités dénuées des moyens techniques et financiers nécessaires pour faire face et résoudre ces problèmes.

\subsection{Recherche de solutions}

En 1989, le Premier Ministre mettait en place une mission ministérielle pour rechercher et mettre en ouvre les solutions adéquates. Parallèlement l'ADEME et les Bureaux d'Etudes concernés travaillent à l'étude des phénomènes et à la mise en place et à la validation d'un programme de sauvegarde technique des installations. Trois actions principales furent alors menées dès 1990, concernant les techniques curatives de nettoyage des puits, les techniques préventives de lutte contre les phénomènes de corrosion-dépôt par injection en fond de puits d'inhibiteur de corrosion et la mise en œuvre d'un nouveau puits en matériau-composite. Les résultats ont été satisfaisants, particulièrement en ce qui concerne la prévention de la corrosion, sa vitesse ayant été divisée par dix.

Aujourd'hui, on peut considérer que l'exploitation de la ressource géothermale basse-énergie, est techniquement maîtrisée. Actuellement, sont opérationnelles avec un taux de disponibilité souvent supérieur à $95 \%, 40$ installations dans le Bassin Parisien, 16 dans le Bassin Aquitain, 4 dans d'autres régions. Elles contribuent pour 170000 Tep/an à l'indépendance énergétique du pays, chauffant plus de 200000 logements, et évitant le rejet annuel de 700000 tonnes de $\mathrm{CO}_{2}$ dans l'atmophère. Le chiffre d'affaires annuel important, se situe aux environs de 500 millions de Francs.

\subsection{Très basse énergie}

Parallèlement la géothermie très basse énergie à partir de PAC installées sur des aquifères situés à moindre profondeur (Température inférieure à $25^{\circ} \mathrm{C}$ ) connaît un développement rapide en France, représentant fin 1994 une contribution énergétique de 40000 Tep.

Si en France, cette contribution énergétique reste très modeste. Ces opérations ont néanmoins globalement permis d’acquérir et de maîtriser des techniques considérées à l'étranger comme des références.

\subsection{Acquits}

A ce titre, rappelons que dès 1975, la D.G.R.S.T. lançait une étude de potentiel géothermique du Bassin Parisien réalisée par ELF et le BRGM, suivirent d'autres régions (Aquitaine, Alsace, Languedoc, etc...) souvent avec l'aide de la Communauté Européenne

Des études thématiques furent réalisées; par exemple: nappe de 0 à $100 \mathrm{~m}$ en Ile de France, de 100 à $1000 \mathrm{~m}$, Trias argileux du Bassin Parisien, etc... En ce sens, études, forages, exploitation et modélisations des réservoirs ont permis en quelques années d'acquérir une meilleure comnaissance des aquifères profonds, de leurs caractéristiques et de leurs potentiels. Concernant les aquiferes peu profonds $(0-100 \mathrm{~m})$ la quasi-totalité du territoire français a été ainsi inventorié.

\section{PERSPECTIVES ACTUELLES DE DÉVELOPPEMENT}

La dernière grande opération géothermique au Dogger a été réalisée en 1986. Depuis trois opérations ont été réalisées, deux en Aquitaine où distribution de chaleur et thermalisme ont été associés et une à Paris (Front de Seine, 1990) sur l'aquifère de l'Albien avec un doublet associé à des PAC assurant chauffage et climatisation selon un technique originale de stockage saisonnier de chaud et de froid.

Il existe certes en région parisienne, une bonne conjonction entre des ressources maintenant connue et des besoins importants, mais le coût actuel des énergies fossiles rend impensable toute opération nouvelle. Néanmoins, pouvoirs publics et exploitants sont confrontés au vieillissement des installations existantes, qui si rien n'est fait, sont condamnées à terme. Les opérations sont ainsi conduites à optimiser installation et exploitation afin de dégager des moyens financiers. A ce titre différents moyens, sont mis en œuvre: les systèmes de protection contre la corrosion, le télésuivi, la modélisation du réservoir et le suivi des caractéristiques du réservoir, etc... Mais également l'amélioration des techniques de nettoyage et le recours à des techniques innovantes de reconditionnement des ouvrages.

L'autre moyen d'optimiser les exploitations existantes consiste à étendre les réseaux de chauffage existants. Une enquête récente montre la possibilité d'un tel développement dans 19 opérations, développement pouvant représenter 30000 logements supplémentaires raccordés et 33000 Tep substituées supplémentaires.

Le programme originel de recherche et développement a été ainsi mis en place, avec l'aide de Bruxelles, sous l'investigation des pétroliers et des géothermiciens pour réparer les tubages endommagés. Cette technique étudiée et mise au point par DRILFLEX, consiste à mettre en place dans le puits une «chaussette» souple en matériaux composites jusqu'à la profondeur voulue, gonflée et polymérisée in situ. Des premiers essais à faible profondeur ont déjà été réalisés. Une expérimentation en vraie grandeur est prévue sur un site géothermal abandonné en région parisienne.

Autre programme original, celui récemment réalisé à Melun l'Almont avec une double innovation : réalisation d'un nouveau forage dévié de production en gros diamètre (13"3/8) équipé d'un tubage acier revêtu intérieurement d'un matériau composite (fibre de verre enrobée de résine époxy), et fonctionnement en triplet avec production dans le nouveau puits et réinjection dans les deux anciens puits dont un réalisé en 1984. L'installation datant de 1969, se trouve ainsi modernisée pour 20 ans, voire plus. 


\section{DIVERSIFICATIONS}

Enfin une diversification des usages devrait permettre d'élargir le champ des réalisations dans les autres structures sédimentaires françaises et de développer des énergies produites localement. Le tableau l, montre les différentes applications envisageables en fonction de la température de la ressource.

Le tableau 2, montre que de telles applications (balnéothérapie, aquaculture, serres, process industriels) sont peu développées en Europe où la ressource géothermale a été essentiellement utilisée pour le chauffage urbain.

Trois exemples de diversification, sont particulièrement intéressants :

- celui de Lamazère, dans le Gers, avec le chauffage de 3,6 ha de serres utilisées pour cultiver des fleurs en grandes quantités. La nappe exploitée est celle des sables de Lussagnet à $1600 \mathrm{~m}$ de profondeur, la température est de $57^{\circ} \mathrm{C}$, le débit en pompage de $180 \mathrm{~m}^{3} / \mathrm{h}$. L'eau est très peu minéralisée $(0,4 \mathrm{~g} / \mathrm{l})$, et n'est pas réinjectée dans le réservoir. Cette installation date de 1982, avec un temps de retour de l'ordre de 7 ans et permet de substituer 1300 Tep/an. L'exploitant actuel prévoit la création de 4 ha supplémentaires de serres en 1996 ;

- celui de Mios Le Tech, en Gironde en bordure du bassin d'Arcachon, avec l'élevage industriel d'esturgeons. La nappe exploitée est celle des grès purbeckiens et de la dolomie de Mano entre 1730 et $1830 \mathrm{~m}$ de profondeur. La tem- pérature est de $76^{\circ}$, le débit en pompage est de $200 \mathrm{~m}^{3} / \mathrm{h}$. L'eau, peu minéralisée $(3,7 \mathrm{~g} / \mathrm{l}))$ autorise un rejet dans le milieu naturel. Le puits est un ancien forage pétrolier beaucoup plus profond, rebouché à 2500 mètres de profondeur et perforation du tubage pour mettre en production le niveau aquifère. L'eau géothermale cède ses calories par le biais d'un échangeur en titane à l'eau de la rivière La Leyre afin d'assurer une température de l'eau des bassins comprise entre 17 et $22{ }^{\circ} \mathrm{C}$. La production annuelle de $30 \mathrm{t}$ d'esturgeons devrait augmenter à $80 \mathrm{t}$. Le temps de retour de l'installation est de 4 ans environ et permet de substituer 4000 Tep/an :

- celui de Saint-Paul-les-Dax, récemment réalisé avec la maîtrise d'œuvre de Geotherma, et dont l'objectif est d'assurer une production combinée Thermalisme-Géothermie. La nappe exploitée est celle du Dano-Paléocène à $1800 \mathrm{~m}$ de profondeur, la température est de $61^{\circ} \mathrm{C}$, le débit en pompage se situe à environ $100 \mathrm{~m}^{3} / \mathrm{h}$. L'eau, faiblement minéralisée (1 g/l), ne nécessite pas de réinjection. Cette installation en cours d'aménagement à un temps de retour prévu de l'ordre de 15 ans, mais ceci sans tenir compte de l'exploitation thermale. L'utilisation purement thermique devrait permettre de substituer $650 \mathrm{Tep} / \mathrm{an}$.

Ces opérations qui ont reçu le soutien de la Communauté Européenne au titre du programme Thermie, constituent une vitrine de démonstration vis-à-vis des marchés étrangers et permettent l'exportation du savoir-faire et des technologies, en particulier vers l'Europe de l'Est, attaches à développer leurs ressources énergétiques locales.

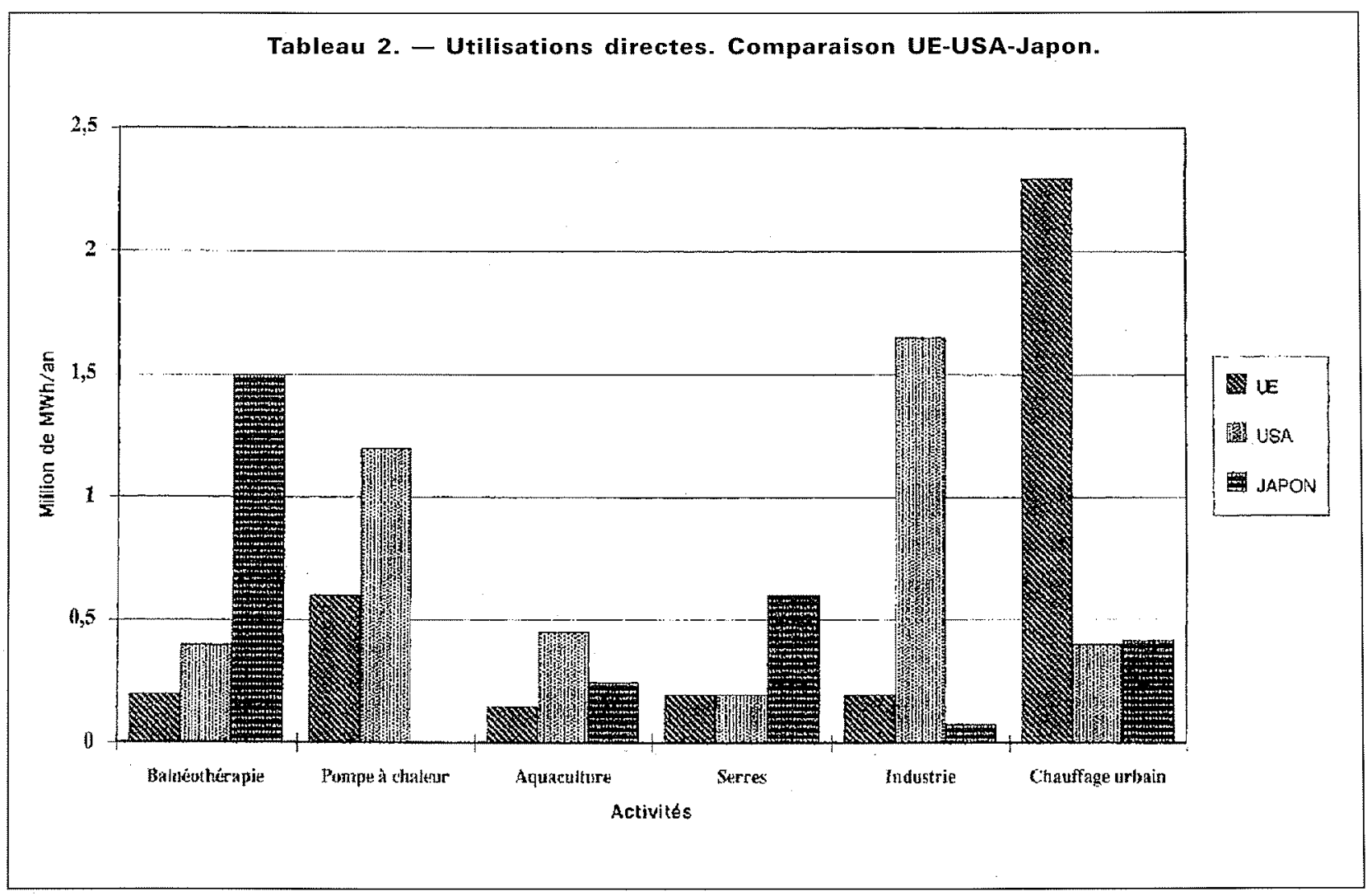




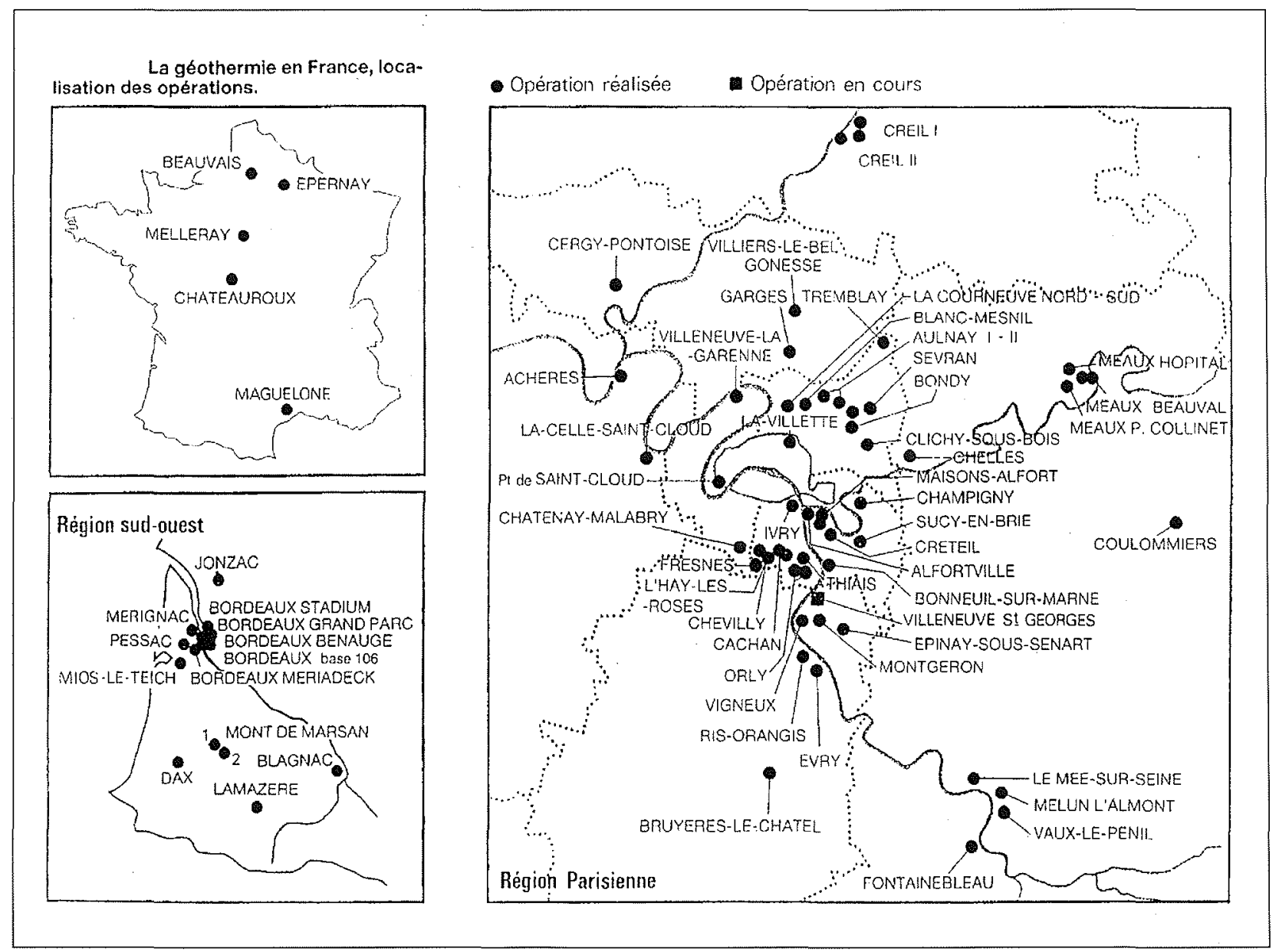

